\title{
El Antropoceno, cuun concepto geológico o cultural, o ambos?
}

\author{
HELMUTH TRISCHLER
}

Cuando Paul J. Crutzen y Eugene F. Stoermer propusieron en 2000 un nuevo tiempo geológico, el Antropoceno, difícilmente previeron la doble connotación del término. Pocos años después, la comunidad geológica comenzó a investigar las evidencias científicas y estableció el Grupo de Trabajo del Antropoceno, que ha examinado posibles marcadores y periodizaciones de la nueva época. Investigadores de otras disciplinas identifican al Antropoceno como concepto cultural. Antropólogos e historiadores, sociólogos y politólogos, filósofos y teólogos, y representantes de otras comunidades académicas han intentado dar sentido a la época de los seres humanos a partir de sus respectivas miradas. Los medios de comunicación también han desarrollado interés en las ramificaciones culturales más amplias del concepto. Este artículo arroja luz sobre el debate acerca del Antropoceno y analiza su trayectoria dual como término geológico y cultural.

Palabras Clave: Antropoceno, Paul J. Crutzen, cultura, historia ambiental, geología, gran aceleración

\section{The Anthropocene, a Geological or a Cultural Concept, or Both?}

When Paul J. Crutzen and Eugene F. Stoermer in 2000 proposed to introduce a new geological time, the Anthropocene, they could hardly foresee the remarkable double carrier to the new term. Only a few years later, the geological community established the Anthropocene Working Group, which started to examine possible periodizations. Scholars from numerous other disciplines have understood the Anthropocene as a cultural concept. Anthropologists and historians, sociologists and political scientists, philosophers and theologians have tried to make sense of the Age of Humans. In addition, the media have developed a deep interest in the broader cultural ramifica-

HELMUTH TRISCHLER

Ludwig-Maximilians-Universität Munchen, Múnich, Alemania h.trischler@deutsches-museum.de Traducción: Amanda Sucar Warrener tions of the concept. The article sheds light on the debate about the Anthropocene and discusses its dual careers as a geological and a cultural term.

KEYWORDS: Anthropocene, Paul J. Crutzen, culture, environmental history, geology, great acceleration 
uando Paul Crutzen y Eugene Stoermer (2000) presentaron por primera vez la idea del Antropoceno en el boletín del Programa Internacional Geósfera-Biósfera (IGBP, por sus siglas en inglés), no podían haber previsto la meteórica trayectoria del Antropoceno. Si lo hubieran sabido, seguramente no habrían publicado su propuesta en un boletín interno, sino en una revista científica prominente para alcanzar a la comunidad científica mundial. Dos años después, Crutzen (2002) rectificó esta omisión cuando presentó su hipótesis en un preciso y sucinto artículo de una página titulado "Geología de la humanidad", en la revista Nature. Los seres humanos, sugirió, se han convertido en una fuerza geológica poderosa, tan es así, que es necesario designar una nueva época geológica para describir con precisión este desarrollo. Esta nueva "época de los seres humanos", el Antropoceno, comenzó con la Revolución industrial a finales del siglo XVIII. La humanidad seguirá siendo una fuerza ambiental predominante durante miles de años (Crutzen, 2002: 23).

El limnólogo Eugene F. Stoermer (1934-2012) ya había comenzado a usar el término Antropoceno de manera informal en la década de 1980, pero fue el químico atmosférico Paul J. Crutzen (1933-), con todo el peso de su reputación como Premio Nobel y descubridor del agujero de ozono, quien de pronto tuvo éxito en la popularización del término. En una conferencia en Cuernavaca, México, en 2000, Crutzen —el "señor Antropoceno"-, se impacientó al escuchar que se mencionaba al Holoceno como la época geológica actual y de manera espontánea exclamó que estamos viviendo en el Antropoceno. Crutzen ha repetido esta historia de un repentino destello de intuición, un momento "eureka" de nuestros días, en múltiples ocasiones (Schwägerl, 2015), y Will Steffen, presente en esa conferencia, lo ha confirmado, y así se ha codificado un atractivo mito fundacional de los orígenes del término (Steffen, Crutzen y Stoermer, 2013: 486).

Crutzen cuenta una historia adicional como parte de este mito fundacional: después de que algunos colegas sugirieran que él debía reclamar la paternidad del término, descubrió que Stoermer lo había elaborado de manera independiente. La autoría conjunta del artículo en el boletín del IGBP, que comenzó todo el debate actual, es un reflejo de esta cocreación.

Hoy, menos de dos décadas después de la publicación del artículo de Crutzen y Stoermer, la discusión acerca de la "época de los seres humanos" se ha 
expandido más allá del ámbito de las ciencias biológicas y geológicas. Se ha convertido - algunos estudiosos se han quejado de esto- en parte de la "cultura popular" (Autin y Holbrook, 2012: 60-61). Investigadores de diversas disciplinas —antropología y teología, geografía y paleografía, arte y literatura - se han involucrado en análisis intensos del Antropoceno. Además, el Antropoceno ya no se limita al ámbito académico y es ampliamente debatido por los medios de comunicación y el público en general. Para dar un ejemplo de hasta qué punto el concepto se ha extendido en el ámbito público, el Deutsches Museum — principal museo de ciencia y tecnología de Alemania - y el Rachel Carson Center for Environment and Society (RCC) crearon la primera gran exposición del mundo sobre el tema: "Bienvenidos al Antropoceno: la tierra en nuestras manos". Se asociaron con la Haus der Kulturen der Welt —Casa de las Culturas del Mundo, centro destacado para las artes y la cultura-, que en 2013 y 2014 dedicó todas sus actividades al Proyecto Antropoceno (Möllers, Schwägerl y Trischler, 2015; Renn y Scherer, 2015; Robin et al., 2014).

¿Qué es tan importante en las discusiones sobre el Antropoceno? Para entender los debates actuales es crucial distinguir entre el Antropoceno en un sentido científico, como un concepto geológico, y el Antropoceno como concepto cultural, en un sentido más amplio. Este artículo arroja luz sobre el controvertido debate acerca del Antropoceno y analiza su trayectoria dual inextricablemente vinculada como un término geológico y como un término cultural. Se argumenta que el debate acerca de la "época de los seres humanos" es una buena oportunidad tanto para superar la división temporal, ontológica, epistemológica e institucional entre naturaleza y cultura que ha dado forma a la visión del mundo occidental desde el siglo XIX, como para explorar nuevas formas de colaboración interdisciplinaria y transdisciplinaria.

\section{El Antropoceno como un concepto geológico}

El debate sobre el Antropoceno como un término geológico es mucho más antiguo. Se remonta a finales del siglo XVIII, precisamente el periodo propuesto por Crutzen y Stoermer como la fecha de comienzo de la nueva época. Seguramente no es casualidad que la idea sea tan antigua como el fenómeno que describe. A medida que la industrialización dejó una marca visible en el mundo, los científicos empezaron a prestar atención. En 1775, el naturalista francés Georges-Louis Leclerc, conde de Buffon, distinguió entre la naturaleza original y la naturaleza civilizada por el ser humano y observó que "toda la faz de la tierra lleva la huella del poder humano" (1778: 237). ${ }^{1}$ En 1864, George P. Marsh describió el poder transformador de los seres humanos, en particular nuestra influencia en la morfología de la superficie de la Tierra. El sacerdote y geólogo italiano Antonio Stoppani sugirió algo muy cercano a la palabra actual cuando escribió acerca de un "antropozoico" en 1873, para subrayar que la era moderna fue dominada por la humanidad. A principios del siglo xx, conforme el impacto de la humanidad se aceleró, estos comentarios se hicieron más frecuentes. En 1913, Vladimir I. Vernadsky (2006) subrayó el papel de los seres humanos como una "fuerza geológica significativa" y su profesor A. P. Pavlov habló de una "era antropogénica". ${ }^{2}$ Sólo dos años después, un joven científico alemán, Ernst Fischer (1915), publicó el artículo "Der Mensch als geologischer Faktor", y en 1922, Robert L. Sherwood

1 Para referencias detalladas, véase Schwägerl (2015). Mauelshagen (en prensa) ofrece un análisis conceptual profundo, mientras la idea de una prehistoria intelectual del término es furiosamente criticada por Hamilton y Grinevald (2015).

2 Vernadsky, como antecesor conceptual del Antropoceno, es discutido por Guillaume (2014). Al existir sólo referencia rusa del trabajo de Pavlov, no fue incluida al final del presente ensayo. 

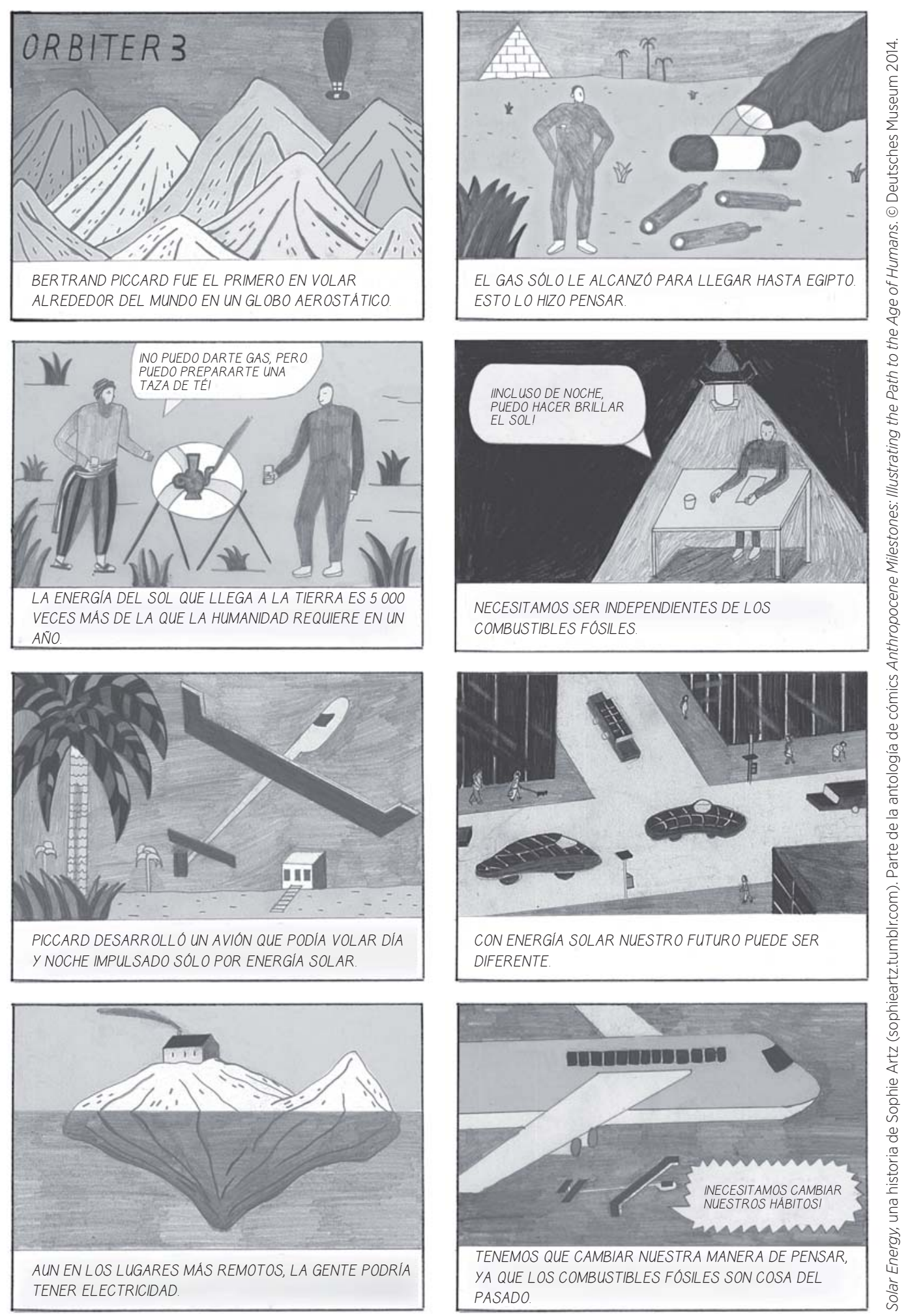
publicó un libro en Londres con un título casi idéntico: El hombre como un agente geológico. A finales del siglo $\mathrm{xx}$, numerosos investigadores anticiparon la idea del Antropoceno. El más destacado fue el biólogo Hubert Markl (1986), quien en la década de 1980 se refirió al Anthropozoikum para describir la era actual.

Cuando Hubert Markl propuso utilizar el término Anthropozoikum no estaba preocupado por la datación del nuevo tiempo geológico: para él lo importante era que este tiempo, marcado en particular por una pérdida masiva de biodiversidad, ya había comenzado. Sin embargo, subestimó los desafíos de su definición. Aunque los defensores del Antropoceno están completamente de acuerdo en que la humanidad, con una tecnología altamente desarrollada, es ahora el actor geológico dominante y continuará siéndolo durante mucho tiempo por venir, hay poco acuerdo sobre la fecha que marca el comienzo de la nueva época. Para Crutzen y Stoermer, en el cambio de siglo, no parecía haber duda de que la datación del Antropoceno debía corresponder con el principio de la industrialización - en específico, con el desarrollo de la máquina de vapor de Watt—, a finales del siglo XVIII. Desde entonces, se han propuesto nueve fechas posibles, la mayoría de las cuales se agrupa en la era moderna, entre 1610 y 1964 (Lewis y Maslin, 2015: 171-180). Tres de estas propuestas han sido prominentes en el debate.

La primera sugerencia se centra en la transición de las sociedades nómadas de cazadores, recolectores y pescadores hacia el establecimiento de sociedades permanentes dedicadas a la agricultura, que se produjo, según los conocimientos actuales, hace alrededor de 11700 años, en el Creciente Fértil en el Cercano Oriente, conocida como la revolución neolítica (Trischler, 2015: 26-27). Este proceso, que duró unos 5000 años, de ninguna manera fue una revolución en el sentido de un cambio súbito en el orden social, pero fue una verdadera transformación: los seres humanos alteraron grandes porciones del paisaje e intervinieron en el acervo genético natural mediante el cultivo de plantas para cosechas y la domesticación de animales en una escala sin precedentes. La "invención" de las sociedades sedentarias, la agricultura y la cría de animales y plantas estaba estrechamente vinculada a las nuevas tecnologías: la cerámica horneada hizo posible almacenar productos agrícolas para su uso posterior. La mejora de las herramientas de piedra y las innovaciones en los métodos de construcción proporcionaron una base para los asentamientos permanentes. La invención del arado hizo posible surcar la tierra para el cultivo y aumentar la productividad.

La revolución neolítica dejó huellas detectables en el registro geológico. Innumerables elementos de evidencia de palinología, arqueología, geología, historia y antropología cultural apoyan la tesis de que las alteraciones humanas en el paisaje de Eurasia comenzaron durante la Edad de Piedra tardía y ganaron un nuevo atributo durante las edades de Bronce y Hierro. Según algunos autores, sólo la intervención humana puede explicar por qué la concentración de dióxido de carbono y metano en la atmósfera presenta un aumento anómalo durante el Holoceno Medio — del siglo vi al siglo III, a. C.-, sustancialmente mayor que las fluctuaciones durante los miles de años previos (Petit et al., 1999).

En segundo lugar, ¿cuál es la evidencia que apoya la datación propuesta por Crutzen que sitúa el comienzo del Antropoceno a finales del siglo XVIII? Hay bibliotecas enteras con literatura sobre si el proceso de industrialización que se originó en Gran Bretaña y transformó por completo a la sociedad, por medio de la tecnología, a lo largo de casi un siglo, puede llamarse propiamente "revolución”. Sin embargo, resultó en la transformación de las sociedades afectadas por la industrialización. Una transformación que de hecho fue bastante revolucionaria en sus efectos. La fuerza impulsora detrás de ella fue una vez más la innovación tecnológica. Tres procesos principales trabajaron juntos: la 
mecanización de la mano de obra; la producción a gran escala y la transformación de la energía creada por la máquina de vapor, y la explotación intensiva y extensiva, la producción y el uso de carbón y hierro.

Los efectos de la industrialización en el medio ambiente y la sociedad fueron aún más esenciales, de largo alcance y duraderos que los de la revolución neolítica. No es casualidad que las discusiones sobre el medio ambiente hayan aumentado de forma constante desde la década de 1830. Los contemporáneos comenzaron a notar que la nueva tecnología alteraba el paisaje y el medio ambiente a profundidad, y con frecuencia incluso su sustento. En el discurso ambiental de los siglos XIX y Xx, la percepción de un cambio en la relación entre la naturaleza y sociedad fue la preocupación principal, además de problemas concretos, por ejemplo, los conflictos sobre la distribución de los recursos naturales, como agua, madera, aire y tierra. En particular extremos fueron los efectos del enorme incremento en el uso de fuentes de energía fósiles que resultaron del uso a gran escala de máquinas alimentadas con carbón. A partir del siglo XIX, ciudades industriales británicas, como Manchester, Glasgow e incluso Londres, adquirieron el dudosamente honorífico título de big smoke - gran humo- (Uekötter, 2009: 20-65). Las locomotoras a carbón y vapor fueron una de las muchas tecnologías basadas en el uso de combustibles fósiles. La rápida propagación del ferrocarril le dio a las industrias del hierro y el acero un gran impulso y aceleró el ritmo de la industrialización. Si revisamos las concentraciones de dióxido de carbono en la atmósfera como un indicador de cambio simple pero significativo, veremos que se incrementó en relación con los niveles preindustriales de 270-275 partes por millón (ppm) a 310 ppm en la segunda mitad del siglo Xx (Petit et al., 1999; Bonneuil y Fressoz, 2016: 15-16).

La tercera datación propuesta, la "gran aceleración" a mediados del siglo Xx, evita usar el término "revolución", pero en vista de la compresión temporal de los acontecimientos, podría describirse mejor así. Como ha demostrado un equipo internacional de investigadores climáticos y del sistema Tierra -Earth system scientists - encabezado por Will Steffen, durante el transcurso de la década de 1950 las curvas de numerosos parámetros cambiaron de una forma lineal a un crecimiento exponencial (Steffen, 2005; Steffen et al., 2015). Esta curva característica, que recuerda a un palo de hockey, se presenta a escala global en fenómenos como el uso de recursos — petróleo crudo, agua y fertilizantes artificiales-, así como la construcción de presas, vehículos, teléfonos y restaurantes McDonald's, e indicadores económicos, por ejemplo, el aumento del turismo internacional, las inversiones extranjeras y el producto nacional bruto. Incluso antes de que comenzara el discurso del Antropoceno, Christian Pfister, historiador económico y ambiental, señaló la mitad del siglo pasado como un importante punto de inflexión, llamado el "síndrome de la década de 1950" (Pfister, 1995). El rápido incremento en el consumo de energía, junto con la motorización masiva de grandes partes del mundo, fue de la mano con el desarrollo de la sociedad de consumo, que se basa en una corriente interminable de innovaciones tecnológicas.

Además de estos contendientes primarios para la periodización del Antropoceno, hay una serie de propuestas adicionales formuladas por equipos individuales de investigadores que a menudo ganan una visibilidad significativa debido a su cobertura en los medios de comunicación masiva. En mayo de 2014, por ejemplo, los hallazgos de un grupo internacional de científicos del Instituto de Investigación del Desierto, en Reno, Nevada, Estados Unidos, fueron noticia en la revista Nature y rápidamente encontraron su camino en los medios de noticias diarias (McConnell et al., 2014). Estos científicos han demostrado que cuando Robert C. Scott y Roald Amundsen llevaron sus expediciones a la Antártida en 1911, en una competencia por ser el primero en 


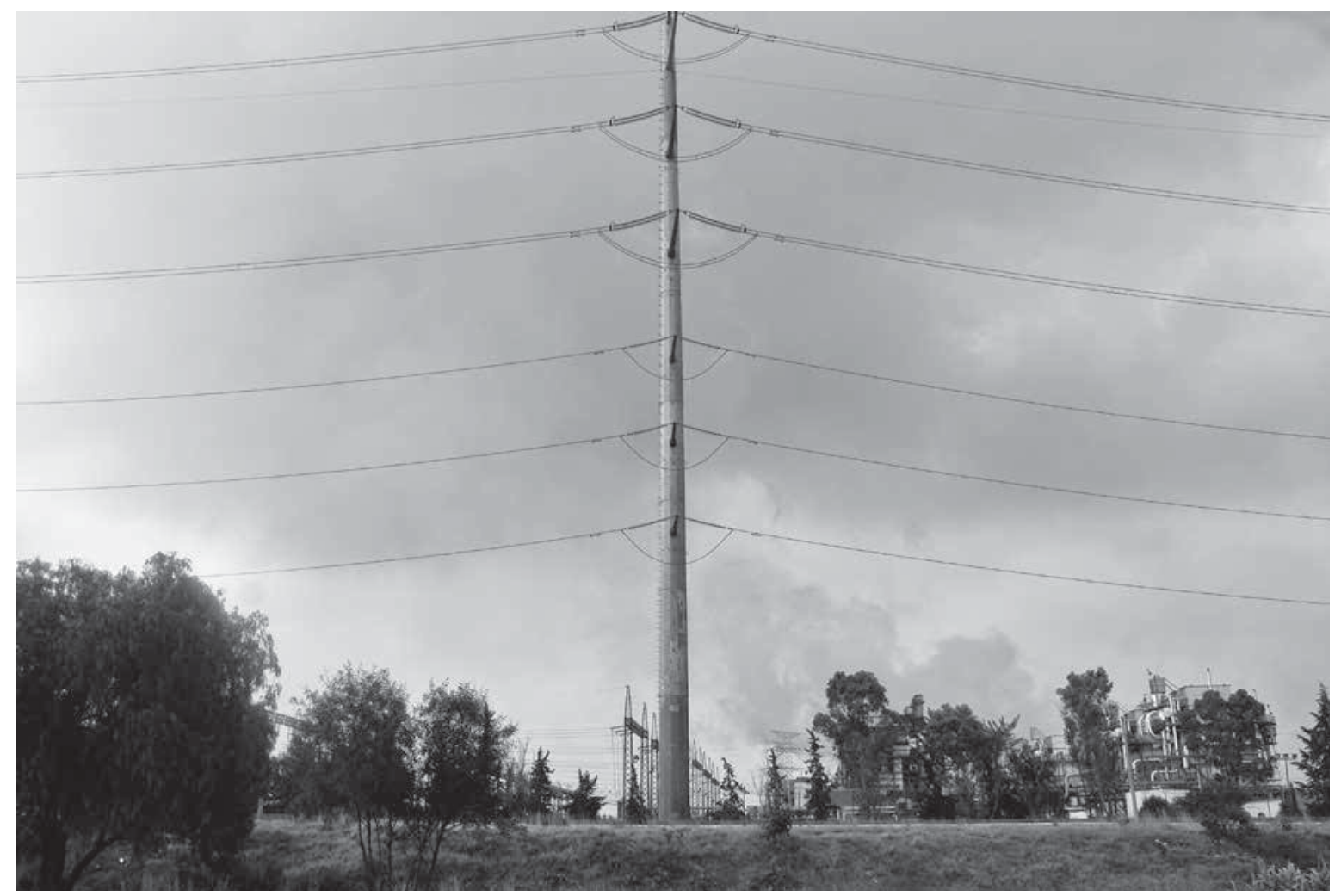

Prometeo Lucero - Termoeléctrica de Tula, Hidalgo, 2010.

llegar al Polo Sur, la nieve y el hielo que se esforzaron en cruzar difícilmente era puro e intacto por la actividad humana. Por el contrario, incluso entonces, en los albores de la Primera Guerra Mundial, la industrialización había dejado sus huellas en la Antártida, el único lugar en la Tierra que parecía haber sido menos impactado por la civilización. A partir de muestras tomadas en 16 ubicaciones distintas en el hielo de la Antártida, este equipo de científicos midió la cantidad anual de contaminación por plomo depositada en la atmósfera entre los años 1600 y 2010. Demostraron que para 1889 la contaminación industrial por plomo, emitida originalmente por la minería y las actividades de fundición, chimeneas de fábricas y motores de combustión, ya se encontraba presente en grandes cantidades, en algunas muestras, dos décadas antes de que los primeros exploradores llegaran al Polo Sur. A finales del siglo XIX, los niveles de plomo depositado en la Antártida fueron más altos que en cualquier momento en el siglo XX. Los datos mostraron que los niveles de contaminación por plomo alcanzaron un máximo alrededor de 1900, que continuó en la década de 1920 antes de volver a caer de nuevo.

En la primavera de 2015, otra propuesta de datación recibió mucha atención en los medios de comunicación masiva. En un artículo, de nuevo publicado en Nature, científicos británicos propusieron que la fecha de comienzo del Antropoceno debería retroceder hasta principios del siglo XVII (Lewis y Maslin, 2015). Argumentaron que las consecuencias de la actividad humana, en particular la colonización de América, estaban ya tan extendidas que un efecto global podría ser identificado, incluso 
antes de la Revolución industrial. Sus análisis de muestras de núcleo de hielo mostraron una caída prominente en los niveles de dióxido de carbono en la atmósfera de la Tierra en 1610. Su causa era un efecto retardado de la llegada de los europeos a América. La colonización del Nuevo Mundo causó la muerte de unos 50 millones de indígenas americanos como resultado de la guerra y las enfermedades introducidas por los europeos, como la viruela. La caída dramática en la población significó que grandes extensiones de tierra que anteriormente se cultivaban quedaran sin explotar y nuevamente se convirtieran en selva. Este aumento de vegetación capturó enormes cantidades de dióxido de carbono de la atmósfera. En 1610, este efecto alcanzó su punto máximo y dejó un rastro marcado en el registro geológico en forma de un descenso en la concentración de dióxido de carbono atmosférico. Que un impacto de ese tipo, relativamente pequeño, sirva hasta la fecha como un marcador del comienzo del Antropoceno es algo severamente cuestionado por muchos geólogos (Zalasiewicz et al., 2015b).

Hace varios años, al Grupo de Trabajo del Antropoceno (AWG, por sus siglas en inglés) se le encomendó la tarea de examinar las evidencias científicas sobre el Antropoceno y presentar a la Subcomisión de Estratigrafía del Cuaternario una propuesta formal basada en sus propias investigaciones estratigráficas. Esta organización reporta a la Comisión Internacional de Estratigrafía, que depende de la Unión Internacional de Ciencias Geológicas. Sólo después de que la propuesta haya pasado con éxito por estos cuatro organismos científicos, la existencia de una nueva división geológica será considerada oficial. Con el fin de ser aceptada en este riguroso proceso examinador, una propuesta debe cumplir tres criterios principales:

1. Una base sincrónica, que es el mismo tiempo de comienzo del Antropoceno en todas partes del mundo, lo que representa un evento decidido de antemano para la definición.
2. Una posición especificada en el registro sedimentario que define esta base sincrónica, es decir, una sección estratotipo y punto de límite global -Global Boundary Stratotype Section and Point, GSSP, por sus siglas en inglés-, conocido como "clavo de oro".

3. Un rango especificado en la jerarquía estratigráfica —etapa, época, periodo, era—.

El AwG presentó un adelanto de su propuesta en agosto de 2016, en el 35 Congreso Internacional de Geología, celebrado en Sudáfrica, en torno a la pregunta: ¿en qué medida las acciones humanas están registradas como señales medibles en los estratos geológicos, y el mundo del Antropoceno es marcadamente distinto a la época estable del Holoceno de los últimos 11700 años que permitieron que la civilización humana se desarrollara? (University of Leicester, Press Office, 2016a). Para responder, están examinando lo que se conoce como firmas estratigráficas, que incluyen una amplia variedad de posibles formas de evidencia, en particular:

- Nuevos materiales, como aluminio elemental, concreto, plástico y partículas esferoidales carbonáceas;

- alteraciones en los procesos de creación de sedimentos, por ejemplo, la eutrofización por los fertilizantes, la captura de sedimentos en las presas, la erosión por la minería o la deforestación;

- señales geoquímicas alteradas en los sedimentos y capas de hielo, por ejemplo, aumentos en los hidrocarburos aromáticos policíclicos, metales pesados, residuos de plaguicidas, aumento del nitrógeno y fósforo;

- presencia en los sedimentos y hielo de radionucleidos naturales y artificiales liberados por las pruebas de bombas nucleares;

- cambios en el ciclo del carbono sobre la base de datos de muestras de núcleos de hielo;

- aumento de la temperatura global y elevación del nivel del mar;

El Antropoceno, ¿un concepto geológico o cultural, o ambos? 
- alteraciones en la biodiversidad, por ejemplo, la desaparición acelerada de especies, la homogeneización debido a la agricultura y la cría de animales, y la suplantación de la biota establecida por las especies invasoras introducidas por humanos.

Un artículo publicado recientemente en la importante revista Science, presenta la respuesta preliminar de los investigadores del Antropoceno a esta cuestión: "el Antropoceno es funcional y estratigráficamente distinto del Holoceno", declara el título del artículo. El grupo encontró marcas estratigráficas que eran o bien completamente nuevas o caían sustancialmente fuera del nivel de variación del Holoceno. Todos estos cambios ocurrían, además, de forma acelerada. Los datos reunidos sugieren que el límite inferior del Antropoceno debe situarse en la mitad del siglo Xx (Waters et al., 2016). Su preferencia sería posicionarlo en la jerarquía estratigráfica como una época, al igual que el Holoceno. Como alternativa, otros investigadores indicaron que la clasificación del Antropoceno como una nueva edad dentro del Holoceno - después del Greenlandian, de 11700 a 8200 años antes de 2000 d. C.; el Northgrippian, de 8200 a 4200, y el Meghalayan, de 4200 al presente (Head y Gibbard, 2015) - también sería una opción.

A finales de agosto de 2016, el AWG presentó su resumen de la evidencia y recomendaciones provisionales. Sus 35 miembros, con una sola abstención, estuvieron de acuerdo en que el concepto de Antropoceno "es geológicamente real" y el fenómeno de escala suficiente para considerarlo parte de la Escala de Tiempo Geológico oficial. La mayoría de miembros del AWG también está de acuerdo en la asignación de una época que signifique que el Holoceno ha terminado (University of Leicester, Press Office, 2016b).

No es seguro que la Subcomisión de Estratigrafía del Cuaternario apruebe sin críticas y propuestas de modificación la sugerencia y la turne al siguiente organismo científico superior, la Comisión Internacional de Estratigrafía. Su presidente, Martin J. Head, ha expresado escepticismo y ha sugerido que las muchas desventajas de la definición de una nueva época geológica superan a las pocas ventajas: la época del Holoceno sería recortada, algo que nunca había sucedido en los 200 años de historia de la geología. Toda la escala de tiempo geológica sería mutilada y convertiría una enorme cantidad de literatura sobre el Holoceno en obsoleta. Pedir a los conservadores expertos estratigráficos que acepten una propuesta de este tipo es una gran solicitud. Las objeciones de los estratígrafos oscilan desde argumentos puramente científicos, como cuestionar la duración a largo plazo de los posibles indicadores de este límite, como la presencia en los sedimentos de los isótopos radioactivos resultantes del uso y las pruebas de armas nucleares, que sólo persistirán durante 100000 años, hasta las reservas morales, como que nombrar un intervalo en la historia de la Tierra por primera vez no sólo haciendo referencia a una sola especie, sino en "honor" de una especie que resultamos ser nosotros mismos, fortalecería la arrogancia que debemos superar si queremos crear un Antropoceno ecológicamente más robusto (Gibbard y Walker, 2013; Finney y Edwards, 2016).

La propuesta puede ser aprobada por la Subcomisión de Estratigrafía del Cuaternario sólo si una mayoría calificada —al menos 60\% de sus miembros - vota a favor. Si no se alcanza esta mayoría, el debate estratigráfico sobre el Antropoceno como una fase distinta en la historia geológica de la Tierra no se terminará. Es importante recordar que el debate sobre el Antropoceno ya no es un tema de interés sólo para los geólogos. Desde hace tiempo ha sido adoptado por otras disciplinas de las ciencias naturales y más allá. Los climatólogos y químicos atmosféricos, ecólogos y oceanógrafos, y muchos otros expertos en ciencias de sistemas relacionados con la 
Tierra tienen una visión más amplia sobre los cambios en el planeta inducidos por el ser humano, que lo que está implícito en la búsqueda de evidencias geológicas en los estratos rocosos. De hecho, el AWG apoya una comprensión del concepto más amplia, que no sólo se relaciona con detectar la influencia humana en la estratigrafía, sino que "refleja un cambio sustancial en el sistema de la Tierra" (Zalasiewicz et al., 2015a: 197; Steffen et al., 2016). En esta mayor comprensión sistémica, el Antropoceno se conecta con el esfuerzo por identificar los "límites planetarios" y el llamado "espacio operativo seguro para la humanidad" (Rockström et al., 2009; Steffen et al., 2015). Si los estratígrafos terminan votando en contra de la propuesta de una nueva época en la historia de la Tierra, el término continuará siendo utilizado por científicos en un sentido sistémico más amplio (Hamilton et al., 2015: 3).

\section{El Antropoceno como un concepto cultural}

La ola de atención puesta en marcha por el reciente artículo del AWG en Science se extendió en los medios de comunicación populares y resaltó cómo el debate sobre el Antropoceno hace tiempo que escapó de los confines de la academia y entró en el discurso público. Incluso antes de la publicación de la versión impresa del artículo, aparecieron textos en línea en medios de noticias como The Guardian, Daily Mail, The Washington Post, Frankfurter Allgemeine Zeitung, Züricher Tagesanzeiger, New Scientist y Scientific American. El mismo día en que se publicó la edición impresa, los principales medios de comunicación de todo el mundo informaron que la comunidad científica había propuesto la designación de una nueva época geológica. El Antropoceno ha captado el interés de los medios de comunicación y se está convirtiendo en una cuestión con implicaciones culturales, que disuelve las fronteras entre ciencia y sociedad.
Ésta es precisamente la mayor importancia del Antropoceno como concepto cultural, que difumina los límites establecidos en muchos ámbitos. Lo más importante, sin embargo, es que abre la posibilidad de liberarnos de dicotomías tradicionales, como naturaleza-cultura, y de redefinir la relación entre el medio ambiente y la sociedad como inextricablemente entrelazados. Bruno Latour va un paso más allá y desafía no sólo la dicotomía moderna de la naturaleza y la cultura, sino su existencia como categorías separadas en primer lugar:

El punto de vivir en la época del Antropoceno es que todos los agentes comparten el mismo destino que cambia de forma. Un destino que no se puede seguir, documentar, deciry representar mediante el uso de cualquiera de los atributos más viejos asociados con la subjetividad o la objetividad. Lejos de tratar de "conciliar" o "combinar" la naturaleza y la sociedad, la tarea política fundamental es, por el contrario, distribuir la agencia tan lejos y de una forma tan diferenciada como sea posible, hasta que hayamos perdido por completo cualquier relación entre estos dos conceptos de objeto y sujeto, que no son más de interés, excepto patrimonial (2014: 17).

Latour concluye con un interesante paralelismo entre el actual debate sobre el papel de los seres humanos como una fuerza geológica y la controversia sobre el estado de los seres humanos en el universo, que Galileo Galilei puso en marcha hace más de 400 años, cuando presentó la tesis de que la Tierra se movía alrededor el sol y cuestionó la concepción establecida del mundo, lo que atrajo la atención de la Inquisición. La tesis del Antropoceno no es que "la Tierra se está moviendo", sino más bien que "la Tierra se mueve" por los seres humanos (Latour, 2014: 3-4). Esta tesis también altera de manera radical nuestra concepción del mundo y convoca a la inquisición de nuestra época, que Latour identifica sobre todo como esos círculos en la política y la 
industria que niegan que los seres humanos son la causa del cambio climático. ${ }^{3}$

La capacidad del Antropoceno para desafiar categorías y límites establecidos es evidente incluso dentro del AGW. Curiosamente, sus debates científicos incluyen no sólo las aportaciones de los geólogos y biólogos - esto es, expertos en estratigrafía y biodiversidad-, sino también de expertos en una amplia variedad de disciplinas. Los 37 miembros de ese grupo de trabajo abarcan investigadores de derecho, antropología e historia, esta última representada por el historiador mundial y ambiental John McNeill y la historiadora de la ciencia Naomi Oreskes. Por primera vez en la historia de la geología, las decisiones sobre la periodización geológica se están discutiendo no sólo entre un grupo selecto de expertos en estratigrafía, sino en un entorno interdisciplinario, en el que las ciencias naturales, las ciencias sociales y las humanidades contribuyen con sus conceptos de tiempo, espacio, producción de evidencia e interpretación. Esto es en sí mismo un escenario muy interesante, que invita, es más, requiere historiadores de la ciencia y la tecnología para reflexionar sobre las consecuencias epistemológicas más amplias de estos marcos interdisciplinarios de producción de conocimiento.

Las voces críticas que expresan dudas sobre el valor analítico del Antropoceno no se limitan a las ciencias geológicas. Como concepto cultural también ha sido desafiado en una amplia variedad de puntos. Algunos críticos dudan acerca de si, como una manera de definir la relación entre naturaleza y cultura, y medio ambiente y sociedad, ofrece algo que las categorías analíticas ya establecidas no pueden. Otros tienen preocupaciones más profundas: temen que el nombramiento de una nueva época geológica que hace referencia a los seres humanos proporcione un estímulo masivo para el ya rampante antropocentrismo y cause que el respeto humano por el valor moral intrínseco de la naturaleza disminuya aún más (LeCain, 2015). ${ }^{4}$ Por otra parte, algunos antropólogos, expertos en "antropos" como una entidad cultural, se sienten incómodos con el término. Para ellos significa toda la humanidad, cuando sólo pequeños grupos de personas en los países industriales son los verdaderos responsables de los problemas ambientales de la modernidad. En lugar de estimular el cambio social y político urgentemente necesario, el término oscurece la responsabilidad concreta al hacer hincapié en las cualidades intrínsecas humanas en lugar de en las opciones que resultan de los intereses capitalistas establecidos (Malm y Hornborg, 2014).

Que no haya malentendidos: el Antropoceno como un concepto cultural no se beneficiaría de nada tanto como de un debate constructivo y crítico acerca de su potencial y eficacia para la redefinición de la relación entre los seres humanos y la naturaleza. Pero el extenso reproche que fortalece fundamentalmente el antropocentrismo no entiende el punto analítico de la convergencia del concepto. El Antropoceno no trata enfáticamente acerca de la afirmación de la dicotomía entre naturaleza y cultura que se ha desarrollado a lo largo de la era moderna, sino sobre poner en duda crítica al antropocentrismo que ha resultado de esto. No es casualidad que el Antropoceno como concepto cultural haya encontrado resonancia particular entre los defensores de los enfoques poshumanistas. La difuminación de las fronteras entre naturaleza y cultura en el Antropoceno otorga agencia sobre actuantes no humanos (Latour, 2014) e identifica una amplia variedad de androides e híbridos en el espacio abierto de formas de existencia poshumanistas.

3 Véase también la conferencia de Bruno Latour, "The Anthropocene and the Destruction of the Image of the Globe". Disponible en línea: <https://www.youtube.com/watch?v=4|6FQN4P1c>. Consultado el 23 de marzo de 2016.

$4 \quad$ Una refutación reflexiva sobre las objeciones comunes al concepto del Antropoceno se encuentra en Schwägerl (2013). 


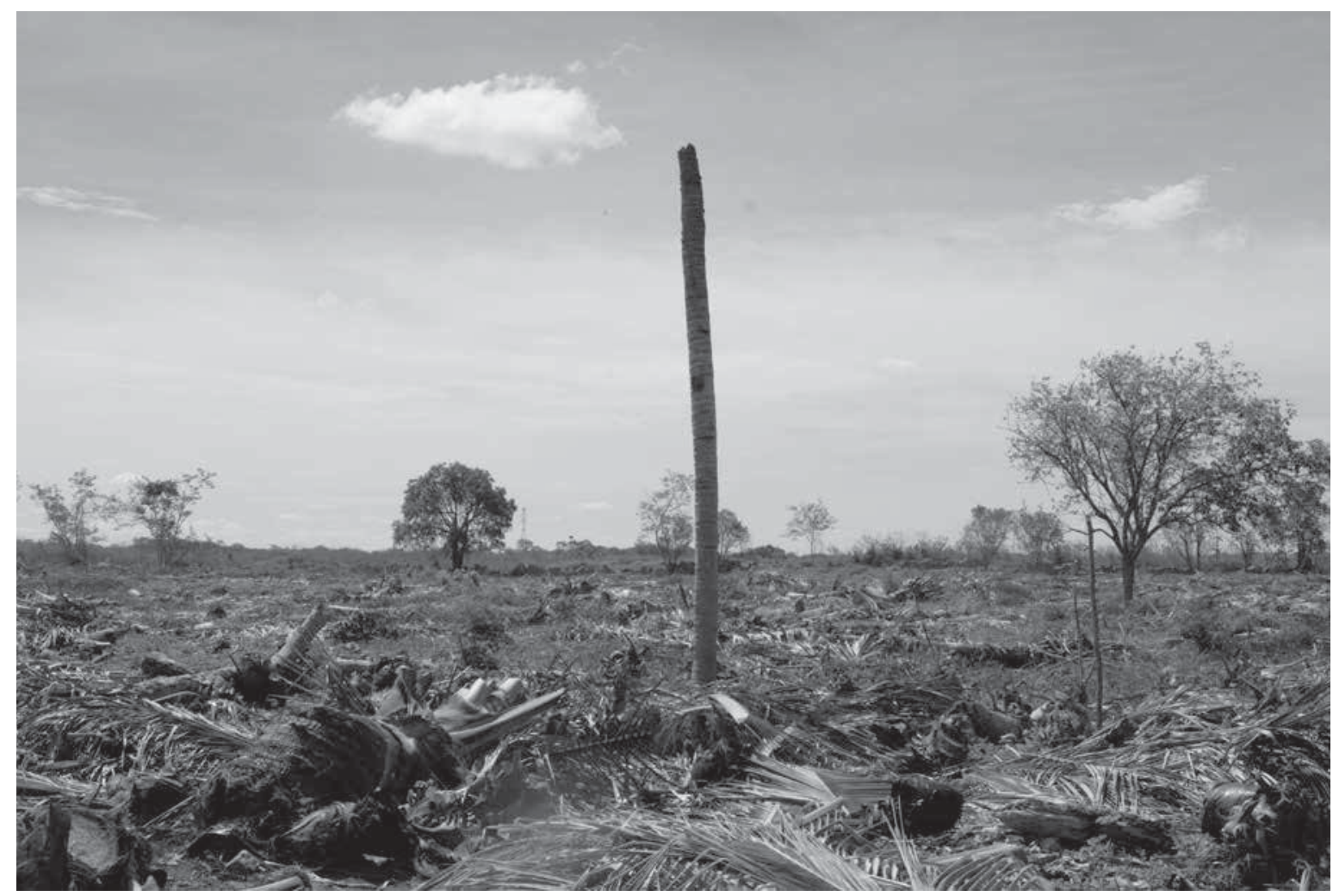

OCtAvIo Hoyos Fábrica de bebidas yucatecas tradicionales de coco y sus derivados. Mérida, Yucatán, junio de 2015.

Concebido de esa manera, el Antropoceno, en última instancia, hace posible un cambio de perspectiva sobre la humanidad y nuestra singularidad entre las otras especies en el planeta. La humanidad se convierte, en palabras de la investigadora literaria y teórica Ursula Heise, pionera poshumanista, en sólo una parte de "las redes de agencias distribuidas que incluyen animales, plantas, sustancias y objetos" del mundo (2015: 40). Heise ofrece un ejemplo. Desde una perspectiva poshumanista, el concepto de Antropoceno significa ser consciente de que...

incluso una simple afirmación de mi propia agencia como "iré a Berlín mañana" depende de una multitud de objetos, sustancias, procesos e instituciones, que yo controlo sólo parcialmente o [no controlo] en absoluto (cafeína, trigo, aspirina, gasolina, caucho, asfalto, carreteras, fabricantes de automóviles, control del tráfico aéreo, horarios, equipaje y redes de teléfonos celulares, por nombrar sólo algunos) (2015: 40).

Donna Haraway, la "gran dama" de los estudios feministas, está de acuerdo y concluye que, lógicamente, hablar del Antropoceno significa hablar del Capitaloceno (2015). En esto se une a las filas de los numerosos politólogos, antropólogos, sociólogos, economistas y filósofos que sugieren que el Antropoceno, como se ha desarrollado desde la industrialización, está estrechamente vinculado con el capitalismo (Head, 2014; Moore, 2016).

Como era de esperarse, el Antropoceno está siendo intensamente debatido en particular entre 
los historiadores. ${ }^{5}$ Como expertos en periodos temporales de la historia humana, los historiadores de todo tipo tienen una conexión directa con los temas que se discuten en el debate del Antropoceno: todas las propuestas para definir el límite temporal del Antropoceno se ocupan de cuestiones centrales estudiadas por la historia de la ciencia, la historia de la tecnología y la historia ambiental. Por lo tanto, es relevante - en especial, para ellos- participar en el debate sobre el Antropoceno. Sobre la cuestión de la definición geológica del Antropoceno, John McNeill y Naomi Oreskes tienen, por así decirlo, un mandato oficial para representar a sus comunidades y la experiencia específica de cada una. Pero para los historiadores que entran en la discusión sobre el Antropoceno como un concepto cultural, la participación en el debate significa nada menos que enfrentar el reto de examinar de manera crítica las historias y discursos establecidos y escudriñar los fundamentos teóricos, metodológicos y conceptuales de la investigación histórica.

Varios historiadores han aceptado este desafío. Por ejemplo, John McNeill recurre a su experiencia como miembro del AWG para narrar la historia del mundo desde 1945 como la del despliegue del Antropoceno, que resultó de la gran aceleración en general y de la expansión masiva del uso de combustibles fósiles en específico, y "más que cualquier otro cambio", inauguró la nueva época (McNeill y Engelke, 2014: 367; 2016). Como historiador ambiental interesado en la circulación de los recursos, McNeill se refiere en particular al uso de energía de combustibles fósiles a gran escala, las emisiones de dióxido de carbono y dióxido de azufre, el uso de agua dulce y el riego, el uso de fertilizantes y la liberación de sustancias químicas tóxicas, por nombrar sólo unos pocos indicadores de cambio ambiental durante la gran aceleración. Para los geólogos en necesidad de un caso claro de clavo de oro, él propone examinar los huesos y dientes de los mamíferos nacidos en las décadas de 1940 y 1950, que por primera vez en la historia incluyen una marca química que resultó de las pruebas de armas nucleares. El tratado de prohibición de pruebas de 1963 comenzó a debilitar la marca de los radionucleidos. Algunos de estos huesos y dientes terminarán en una capa de sedimento que distingue la mitad del siglo XX de todo lo anterior y todo lo que vino después.

La ciencia política, la antropología, la sociología y la filosofía, junto con la historia, son las comunidades de las humanidades y las ciencias sociales en las que el Antropoceno como concepto cultural está siendo debatido con más intensidad. Un breve resumen como éste no puede discutir de manera adecuada todos los aspectos de un discurso tan amplio y multifacético. Como un indicio de su importancia, hay varias revistas nuevas dedicadas al tema: Elementa: Science of the Anthropocene y Anthropocene, ambas en representación exclusiva de las ciencias naturales, y The Anthropocene Review, que se centra además en las perspectivas de las humanidades y las ciencias sociales. Para no distorsionar el panorama, es necesario añadir que otras comunidades académicas también están involucradas en las discusiones sobre el Antropoceno, por ejemplo, el derecho (Kersten, 2013; Eagle, 2016) y la religión (Szerszynski, 2006; Deane-Drummond et al., en prensa). Tampoco se limita a las discusiones teóricas en las artes, la estética y la teoría cultural. Artistas y arquitectos están empezando a responder al imperativo geológico en su práctica y discuten la importancia del término para su obra (Davis y Turpin, 2015; Turpin, 2013).

El Antropoceno también se ha establecido en otro ámbito basado en la práctica, como la educación y la enseñanza, en el que ha sido acogido como un poderoso instrumento para poner a prueba

5 Para una discusión detallada sobre el desafío del Antropoceno para la historia de la ciencia, la tecnología y el medio ambiente, véase Trischler (2016). 
nuevos métodos de educación ambiental (Leinfelder, 2013; Lloro-Bidart, 2015). Las instituciones dedicadas a la difusión del conocimiento, museos y centros de exposiciones, a menudo inspirados en la innovadora exposición en el Deutsches Museum, han descubierto que el tema ofrece una forma de ampliar su repertorio conceptual y temático (Robin et al., 2014). En la actualidad, varias docenas de exposiciones sobre el Antropoceno se están preparando en todo el mundo. A partir de una de estas iniciativas, el Proyecto Antropoceno de la Haus der Kulturen der Welt, en Berlín, ha surgido un ambicioso esfuerzo sin precedentes para crear un plan de estudios interdisciplinario para el Antropoceno (The Anthropocene Curriculum, 2015). ${ }^{6}$ Algunos estudiosos de las humanidades, representados prominentemente por Jürgen Renn, coorganizador del plan de estudios del Antropoceno y director del Instituto Max Planck de Historia de la Ciencia, ven el debate sobre el Antropoceno como una oportunidad para reestructurar nuestro sistema de conocimiento de manera que sea fundamentalmente interdisciplinario y reflexivo, así como radicalmente orientado al diálogo con el público (Renn et al., 2015). Sus interdisciplinariedad y transdisciplinariedad inherentes son otra forma en la que el Antropoceno revela su poder para difuminar fronteras y trascender barreras.

El concepto "Antropoceno" implica algo más que un intercambio de ideas científicas, es más bien un sitio en el que se renegocian posiciones éticas fundamentales. La gran controversia acerca del "buen Antropoceno", comenzada por Erle C. Ellis (2012), encapsula esto como ningún otro tema en el debate. Ellis, un geógrafo y ecólogo del paisaje estadounidense, ve que "los únicos límites para la creación de un planeta del que las generaciones futuras estén orgullosas son nuestra imaginación y nuestros sistemas sociales". Continúa: "al avanzar hacia un mejor Antropoceno, el medio ambiente será lo que hagamos de él" (2015: 54).
La idea del "buen Antropoceno" ha provocado críticas generalizadas de aquellos que temen que allana el camino para conceptos cuestionables de geoingeniería (Latour, 2012; 2015; Hamilton, 2015; Hourdequin, en prensa). Los críticos señalan que hace varios años, nada menos que el "padre" del Antropoceno, Paul J. Crutzen, desesperado por los efectos del cambio climático antropogénico, publicó la sugerencia de detener el calentamiento global mediante la inyección de 1.5 millones de toneladas de partículas de dióxido de azufre a la atmósfera con el fin de reflejar la luz del sol (Crutzen, 2006). Crutzen ha sido muy criticado por esto. Como consecuencia de esa conversación sobre intervenciones tecnológicas cuando las consecuencias a largo plazo para el clima y la Tierra son completamente desconocidas, la idea del Antropoceno ha dejado a muchos un mal sabor de boca (Hamilton, 2013).

Ellis pertenece también a un grupo de 18 autores que publicó un "Manifiesto ecomodernista" en abril de 2015. Se basa en la convicción de que "el conocimiento y la tecnología, aplicados con sabiduría, podrían permitir un buen, o incluso grandioso, Antropoceno" (Asafu-Adjaye et al., 2015). Un buen Antropoceno "exige que los seres humanos utilicen sus crecientes poderes sociales, económicos y tecnológicos para mejorar la vida de las personas, estabilizar el clima y proteger el mundo natural" (Asafu-Adjaye et al., 2015). Entre los autores del manifiesto están Michael Shellenberger y Ted Nordhaus, fundadores del Breakthrough Institute - un centro de estudios estadounidense con estrechos vínculos con la industria-, quienes han publicado libros sumamente polémicos que predicen el fin del movimiento ambiental, se oponen a las negociaciones climáticas y defienden el uso constante de la energía nuclear con la finalidad de proteger el clima (Shellenberger y Nordhaus, 2005; Nordhaus y Shellenberger, 2007).

6 Véase también Renn y Scherer (2015).

El Antropoceno, ¿̇un concepto geológico o cultural, o ambos? 


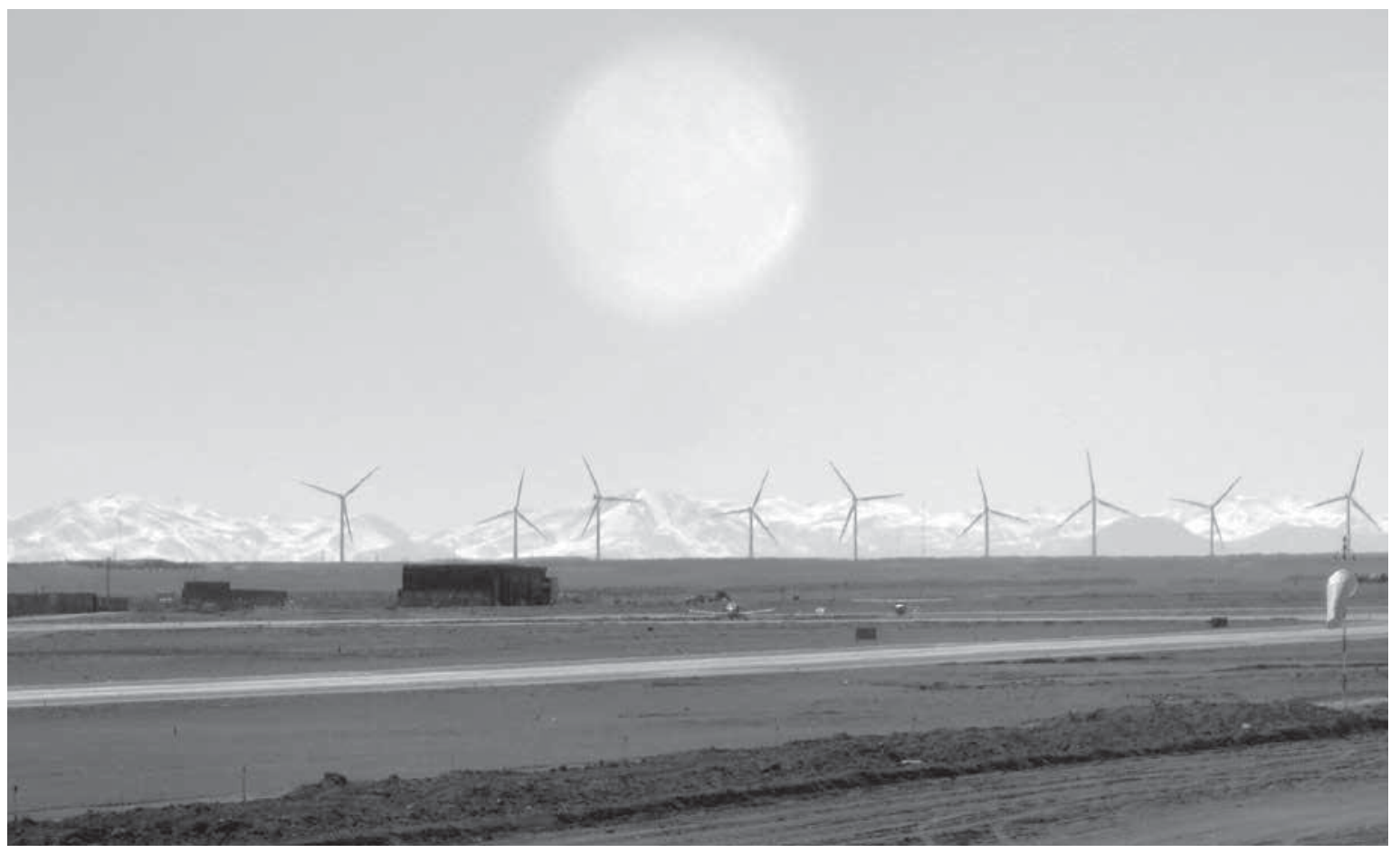

Gwennhael Huesca Reyes • Parque eólico Valle de los Vientos, Calama, Chile, abril de 2015.

De manera característica, el Breakthrough Institute también pide nuevas narrativas, que proporciona en sus propias historias revisionistas. Es particularmente controvertido el intento de recalcular los costos acumulados de la energía nuclear a lo largo del tiempo mientras que confía en tesis extremadamente dudosas, como "no hay una tendencia inherente en el aumento de los costos de la energía nuclear" y los costos de reactores nucleares "incrementan antes de caer", que el Instituto ofrece como consejos para legisladores para justificar la construcción de nuevos reactores en lugar de alejarse de la energía nuclear (Lovering, Yip y Nordhaus, 2016).

El debate cultural sobre el Antropoceno se interesa nada menos que en las cuestiones más centrales de nuestra sociedad: ¿cómo será el futuro? ¿Cómo debemos hacer negocios, trabajar y vivir? ¿Qué papel tendrá la tecnología en esto? ¿Qué formas de producción y comunicación del conocimiento son adecuadas para el Antropoceno? Por último, ¿qué narrativas necesitamos para comprender mejor el papel planetario de los seres humanos como actores que afectan a todo el sistema de la Tierra? Esto es lo que hace que la discusión sea tan fascinante y tan relevante para hoy y mañana.

\section{Perspectiva}

Después de pocos años de haber aparecido en escena, el término Antropoceno se ha extendido ampliamente en la academia y ha ganado una fuerte adherencia social. El concepto es tan refutado como generativo. Ha estimulado una variedad de "narrativas y visiones del mundo sobrepuestas y conflictivas", muchas de ellas cargadas con afirmaciones de largo alcance (Moore, 2015: 1). Por ejemplo, según Latour, el Antropoceno es nada menos que "el concepto 
filosófico, religioso, antropológico y, como veremos, político más decisivo producido hasta el momento como una alternativa a las nociones mismas de 'moderno' y 'modernidad'” (Latour, 2013: 77). Para sus compatriotas Christophe Bonneuil y Jean-Baptiste Fressoz, el "choque del Antropoceno" pide un "reencuentro [conceptual] del tiempo humano (histórico) y del tiempo de la Tierra (geológico)" para superar la división temporal, ontológica, epistemológica e institucional entre la naturaleza y la cultura, el medio ambiente y la sociedad, que ha dado forma a la visión del mundo occidental desde el siglo XIX. En otras palabras, las "narrativas grandiosas e impotentes sobre la modernidad" (Bonneuil y Fressoz, 2016: 33, 290). De hecho, la reintegración de lo no humano en narrativas históricas y ontologías antropológicas es tal vez el común denominador con el que la mayoría de los investigadores que trabajan sobre el Antropoceno estarían de acuerdo.
Como se muestra, el concepto ya ha catalizado un paisaje floreciente de la investigación innovadora en muchos campos académicos. Sin embargo, esto es sólo el comienzo de una trayectoria intelectual que sondeará el potencial heurístico y analítico del concepto. Tomará muchos años, si no es que décadas, para que el concepto de Antropoceno despliegue su poder transformador tanto para las ciencias naturales como para las humanidades. Ya en la actualidad, el concepto se ha convertido en una "zona comercial" para la colaboración en todo el espectro académico (Moore, 2015: 2), que a menudo estimula una "gran interdisciplinariedad" entre los científicos de la naturaleza y los humanistas. Hay mucho más que ganar al aventurarse fuera de límites disciplinarios establecidos y la exploración de nuevas formas de colaboración tanto interdisciplinaria como transdisciplinaria. $\mathbb{D}$

\section{Bibliografía}

Anthropocene Curriculum, The, 2015. Disponible en línea: <http://www.anthropocene-curriculum.org/>. Consultado el 23 de marzo de 2016. Asafu-Adjaye, John et al., 2015, "An Ecomodernist Manifesto". Disponible en línea: <http://www.ecomodernism.org/manifesto-english/>.

Autin, Whitney J. y John M. Holbrook, 2012, "Is the Anthropocene an Issue of Stratigraphy or Pop Culture?", en GSA Today, vol. 22, núm. 7, pp. 60-61. Bonneuil, Christophe y Jean-Baptiste Fressoz, 2016, The Shock of the Anthropocene: The Earth, History, and Us, Verso Books, Nueva York.

Crutzen, Paul J., 2002, "Geology of Mankind”, en Nature, vol. 415, núm. 6867, p. 23.

__, 2006, "Albedo Enhancement by Stratospheric Sulfur Injections: A Contribution to Resolve a Policy Dilemma?”, en Climatic Change, núm. 77, pp. 211-220.

Crutzen, Paul J. y Eugene F. Stoermer, 2000, "The 'Anthropocene'”, en Global Change Newsletter, núm. 41, pp. 17-18.

Davis, Heather y Etienne Turpin (eds.), 2015, Art in the Anthropocene: Encounters among Aesthetics, Politics, Environments and Epistemologies, Open Humanities Press, Londres.

Deane-Drummond, Celia, Markus Vogt, Sigurd Bergmann y Michael Northcott (eds.), en prensa, Religion and the Anthropocene, Wipf and Stock/Cascade Books, Eugene.

Eagle, Josh, 2016, "Climate Change and the Confluence of Natural and Human History: A Lawyer's Perspective", en Robert Emmett y Thomas Lekan (eds.), Whose Anthropocene? Revisiting Dipesh Chakrabarty's "Four Theses", Rachel Carson Center for Environment and Society (Perspectives 2016, 2), Múnich, pp. 21-26.

Ellis, Erle, 2012, “The Planet of No Return”, en Breakthrough Journal. Disponible en línea: <http://thebreakthrough.org/index.php/journal/ past-issues/issue-2/the-planet-of-no-return>. Consultado el 28 de febrero de 2016.

_ 2015, "The Used Earth. Embracing Our History as Transformers", en Nina Möllers, Christian Schwägerl y Helmuth Trischler (eds.), Welcome to the Anthropocene. The Earth in Our Hands, Deutsches Museum Verlag, Múnich, pp. 52-55.

Finney, Stanley y Lucy Edwards, 2016, “The 'Anthropocene' Epoch: Scientific Decision or Political Statement?”, en GSA Today, vol. 26, núm. 3, pp. 4-10. 
Fischer, Ernst, 1915, "Der Mensch als geologischer Faktor", en Zeitschrift der Deutschen Geologischen Gesellschaft, vol. 67, pp. 106-149.

Gibbard, Philip L. y Michael Walker, 2013, "The Term 'Anthropocene' in the Context of Formal Geological Classification”, The Geological Society of London, Special Publication 395, pp. 29-37.

Guillaume, Bertrand, 2014, “Vernadsky's Philosophical Legacy: A Perspective from the Anthropocene”, en The Anthropocene Review, vol. 1, núm. 2, pp. 137-146.

Hamilton, Clive, 2013, Earthmasters. The Dawn of the Age of Climate Engineering, Yale University Press, New Haven.

__ 2015, "The Theodicy of the 'Good Anthropocene'”, en Environmental Humanities, núm. 7, pp. 233-238.

Hamilton, Clive y Jacques Grinevald, 2015, "Was the Anthropocene Anticipated?", en The Anthropocene Review, vol. 2, núm. 1, pp. 59-72.

Hamilton, Clive et al., 2015, "Thinking the Anthropocene“, en Clive Hamilton, Christophe Bonneuil y François Gemenne (eds.), The Anthropocene and the Global Environmental Crisis. Rethinking Modernity in a New Epoch, Routledge, Londres, pp. 1-13.

Haraway, Donna, 2015, “Anthropocene, Capitalocene, Plantationocene, Chthulucene: Making Kin”, en Environmental Humanities, núm. 6, pp. 159-165. Head, Lesley, 2014, “Contingencies of the Anthropocene. Lessons from the 'Neolithic'”, en The Anthropocene Review, vol. 1, núm. 2, pp. 1-13.

Head, Martin J. y Philip L. Gibbard, 2015, "Formal Subdivision of the Quaternary System/Period: Past, Present, and Future”, en Quaternary International, núm. 383, pp. 4-35.

Heise, Ursula, 2015, "Posthumanism. Reimagining the Human", en Nina Möllers, Christian Schwägerl y Helmuth Trischler (eds.), Welcome to the Anthropocene. The Earth in Our Hands, Deutsches Museum Verlag, Múnich, pp. 38-42.

Hourdequin, Marion, en prensa, "Ethics, Adaptation, and the Anthropocene", en Andrew Light y Kenneth Shockley (eds.), Ethics in the Anthropocene, MIT Press, Cambridge.

Kersten, Jens, 2013, “The Enjoyment of Complexity: A New Political Anthropology for the Anthropocene?", en Helmuth Trischler (ed.), Anthropocene: Exploring the Future of the Age of Humans, Rachel Carson Center for Environment and Society (Perspectives 2013, 3), Múnich, pp. 39-55.

Latour, Bruno, 2012, "Love your Monsters. Why We Must Care for Our Technologies as We Do Our Children", en Breakthrough Journal. Disponible en línea: <http://thebreakthrough.org/index.php/journal/past-issues/issue-2/love-your-monsters>. Consultado el 28 de febrero de 2016.

__, 2013, "Facing Gaia. A New Enquiry into Natural Religion", serie de ponencias presentadas en Gifford Lectures, Universidad de Edimburgo, Edimburgo, 18 a 28 de febrero. Disponible en línea: <http://www.ed.ac.uk/humanities-soc-sci/news-events/lectures/ gifford-lectures/archive/series-2012-2013/bruno-latour>. Consultado el 24 de marzo de 2016.

__. 2014, "Agency at the Time of the Anthropocene", en New Literary History, vol. 45, núm. 1, pp. 1-18.

_ 2015, "Telling Friends from Foes in the Time of the Anthropocene", en Clive Hamilton et al. (eds.), The Anthropocene and the Global Environmental Crisis. Rethinking Modernity in a New Epoch, Routledge, Londres, pp. 145-155.

LeCain, Timothy J., 2015, "Against the Anthropocene: A Neo-Materialist Perspective", en International Journal of History, Culture, and Modernity, vol. 3, pp. 1-28.

Leclerc, Georges-Louis, conde de Buffon, 1778, Les époques de la nature, L'Imprimerie Royale, París.

Leinfelder, Reinhold, 2013, “Assuming Responsibility for the Anthropocene: Challenges and Opportunities in Education”, en Helmuth Trischler (ed.), Anthropocene: Exploring the Future of the Age of Humans, Rachel Carson Center for Environment and Society (Perspectives 2013, 3), Múnich, pp. 9-27.

Lewis, Simon L. y Mark A. Maslin, 2015, “Defining the Anthropocene”, en Nature, vol. 519, núm. 2, pp. 128-146.

Lloro-Bidart, Teresa, 2015, "A Political Ecology of Education in/for the Anthropocene”, en Environment and Society, núm. 6, pp. 128-148.

Lovering, Jessica R., Arthur Yip y Ted Nordhaus, 2016, "Historical Construction Costs of Global Nuclear Power Reactors", en Energy Policy, vol. 91, pp. 371-382.

Malm, Andreas y Alf Hornborg, 2014, "The Geology of Mankind? A Critique of the Anthropocene Narrative", en The Anthropocene Review, vol. 1, núm. 1, pp. 62-69.

Markl, Hubert, 1986, Natur als Kulturaufgabe. Über die Beziehung des Menschen zur lebendigen Natur, Deutsche Verlags-Anstalt, Stuttgart.

Marsh, George P., 1864, Man and Nature, Scribner, Nueva York.

Mauelshagen, Franz, en prensa, '“Geology of Mankind': The History of an Idea and Why it Matters", en Celia Deane-Drummond, Markus Vogt, Sigurd Bergmann y Michael Northcott (eds.), Religion and the Anthropocene, Wipf and Stock/Cascade Books, Eugene.

McConnell, J. R. et al., 2014, "Antarctic-Wide Array of High-Resolution Ice Core Records Reveals Pervasive Lead Pollution Began in 1889 and Persists Today”, en Scientific Reports, vol. 4, núm. 5848, pp. 1-5. 
McNeill, John R. y Peter Engelke, 2014, "Into the Anthropocene: People and Their Planet", en Akira Iriye (ed.), Global Interdependence: The World after 1945, Harvard University Press, Cambridge, pp. 365-533.

—, 2016, The Great Acceleration, Harvard University Press, Cambridge.

Moore, Amelia (coord.), 2015, “The Anthropocene. A Critical Exploration”, en Environment and Society: Advances in Research, vol. 6, núm. 1, pp. 1-3. Moore, Jason W. (ed.), 2016, Anthropocene or Capitalocene? Nature, History, and the Crisis of Capitalism, PM Press, Oakland.

Möllers, Nina, Christian Schwägerl y Helmuth Trischler (eds.), 2015, Welcome to the Anthropocene: The Earth in Our Hands, Deutsches Museum Verlag, Múnich.

Nordhaus, Ted y Michael Shellenberger, 2007, Break Through: From the Death of Environmentalism to the Politics of Possibility, Houghton Mifflin Harcourt, Boston.

Petit, Jean-Robert et al., 1999, "Climate and Atmospheric History of the Past 420000 Years from the Vostok Ice Core, Antarctica", en Nature, vol. 399, núm. 6735, pp. 429-436.

Pfister, Christian, 1995, Das 1950er Syndrom. Der Weg in die Konsumgesellschaft, Paul Haupt, Berna.

Renn, Jürgen y Bernd Scherer (eds.), 2015, Das Anthropozän. Zum Stand der Dinge, Matthes und Seitz, Berlín.

Renn, Jürgen et al., 2015, "Knowledge in the Anthropocene", Max Planck Society Research Report, Berlín.

Robin, Libby et al., 2014, "Three Galleries of the Anthropocene”, en The Anthropocene Review, vol. 1, núm. 3, pp. 207-224.

Rockström, Johan, et al., 2009, "A Safe Operating Space for Humanity", en Nature, núm. 461, pp. 472-475.

Schwägerl, Christian, 2013, "Neurogeology: The Anthropocene's Inspirational Power", en Helmuth Trischler (ed.), en Anthropocene Envisioning the Future of the Age of Humans, Rachel Carson Center for Environment and Society, Múnich, pp. 29-38.

—, 2015, "A Concept with a Past", en Nina Möllers, Christian Schwägerl y Helmuth Trischler (eds.), Welcome to the Anthropocene. The Earth in Our Hands, Deutsches Museum Verlag, Múnich, pp. 128-129.

Shellenberger, Michael y Ted Nordhaus, 2005, The Death of Environmentalism: Global Warming Politics in a Post-Environmental World, The Breakthrough Institute, Oakland.

Sherwood, Robert L., 1922, Man as a Geological Agent: An Account of His Action on Inanimate Nature, H. F. and G. Witherby, Londres.

Steffen, Will, 2005, Global Change and the Earth System. A Planet Under Pressure, Birkhäuser, Stuttgart.

Steffen, Will, Paul J. Crutzen y Eugene F. Stoermer, 2013, “The 'Anthropocene' (2000)”, en Libby Robin, Sverker Sörlin y Paul Warde (eds.), The Future of Nature. Documents of Global Change, Yale University Press, New Haven, p. 486.

Steffen, Will et al., 2015, “Planetary Boundaries: Guiding Human Development on a Changing Planet”, en Science, vol. 347, núm. 6223.

_- 2016, "Stratigraphic and Earth System Approaches to Defining the Anthropocene", en Earth's Future, vol. 4, pp. 324-345.

Stoppani, Antonio, 1873, Corso di Geologia, Bernadoni e Brigola, Milán.

Szerszynski, Bronislaw, 2006, Nature Technology and the Sacred, The Free Press, Londres.

Trischler, Helmuth, 2015, "The Anthropocene from the Perspective of the History of Technology", en Nina Möllers, Christian Schwägerl y Helmuth Trischler (eds.), Welcome to the Anthropocene. The Earth in Our Hands, Deutsches Museum Verlag, Múnich, pp. 25-29.

_ 2016, "The Anthropocene. A Challenge for the History of Science, Technology, and the Environment", NTM. Zeitschrift für Geschichte der Wissenschaften, Technik und Medizin, vol. 24, núm. 3, pp. 309-335.

Turpin, Etienne (ed.), 2013, Architecture in the Anthropocene: Encounters among Design, Deep Time, Science and Philosophy, Open Humanities Press, Ann Arbor.

Uekötter, Frank, 2009, The Age of Smoke. Environmental Policy in Germany and the United States, 1880-1970 The Age of Smoke. Environmental Policy in Germany and the United States, 1880-1970, University of Pittsburgh Press, Pittsburgh.

University of Leicester, Press Office, 2016a, “The Anthropocene: Hard Evidence for a Human-Driven Earth”, 7 de enero. Disponible en línea: <https://www2.le.ac.uk/offices/press/press-releases/2016/january/the-anthropocene-hard-evidence-for-a-human-driven-earth>. Consultado el 30 de marzo de 2016.

__, 2016b, "Media note: Anthropocene Working Group (AWG)", 29 de agosto. Disponible en línea: <https://www2.le.ac.uk/offices/press/ press-releases/2016/august/media-note-anthropocene-working-group-awg>. Consultado el 3 de noviembre de 2016.

Vernadsky, Vladimir I., 2006, Essays on Geochemistry and the Biosphere, Synergetic Press, Santa Fe.

Waters, Colin N. et al., 2016, "The Anthropocene is Functionally and Stratigraphically Distinct from the Holocene”, en Science, vol. 351, núm. 6269.

Zalasiewicz, Jan et al., 2015a, "When did the Anthropocene Begin? A Mid-Twentieth Century Boundary Level is Stratigraphically Optimal”, en Quaternary International, vol. 383, pp. 196-203.

, 2015b, "Colonization of the Americas, 'Little Ice Age' Climate, and Bomb-Produced Carbon: Their Role in Defining the Anthropocene", en The Anthropocene Review, vol. 2, núm. 2, pp. 117-127. 